\title{
Communications
}

\section{Technological Thinking, Communication and Behavior of Androids}

\author{
Evgeniy Bryndin \\ Research Centre "Natural Informatic", National Supercomputer Technological Platform, Novosibirsk, Russia
}

Email address:

bryndin15@yandex.ru

\section{To cite this article:}

Evgeniy Bryndin. Technological Thinking, Communication and Behavior of Androids. Communications. Vol. 6, No. 1, 2018, pp. $13-19$. doi: $10.11648 /$ j.com.20180601.13

Received: January 23, 2018; Accepted: February 3, 2018; Published: February 27, 2018

\begin{abstract}
Psychologists of a number of the American universities, including Harvard and Yale, showed that creative thinking depends on the functional connectivity of three different networks of a brain. We classify them as network feeling knowledge, network of imitative thinking and behavioural network. Technological thinking, communication and behavior of androids can be realized by means of hierarchically coherent associative and communicative networks. The hierarchy is under construction of intrinsic network of language thinking of the highest level and associative and communicative networks of the lowest level: imitative thinking and behavior. The intrinsic network of language thinking of the highest level is the recursion neuronet with a reinforcement and cells of memory. The intrinsic network of language thinking realizes technological thinking and communication of the android and initiates work of associative and communicative networks of the lowest level: imitative thinking and behavior. Associative and communicative the network of imitative thinking realizes the formalized actions [1]. Associative and communicative the network of behavior adapts and will organize physical operations of the robot [2]. Adaptation of behavior is defined by the current parameters of semantic network of physical operations of the robot.
\end{abstract}

Keywords: Technological Thinking, Android, Adaptive Behavior, Intrinsic Neuronet, Smart Drugstores

\section{Methodology of Technological Thinking of the Android}

There are several approaches to realization of technological thinking of androids. For example, on the basis of formal language of the description and processing of sense of texts in a natural language [3]. Or on the basis of imitative thinking [4]. Let's consider technological thinking of the android on the basis of intrinsic language thinking of the person.

\subsection{Language Communicative Logic}

Informational unit of language thinking and speech communication of the person is the word. The word calls the representative or a visible material world, either the virtual world, or the abstract world.

The language logic of thinking is a process of communicative merge of words in phrases, phrases in offers, offers in judgments. Communicative merge of words in phrases is carried out according to the scheme of signs, phrases in offers is carried out on a situational to the scheme of signs, offers in judgments on the situational scheme.

Communicative merge of phrases in offers, offers in judgments is carried out on attributes of informational need for subject domain of knowledge. Received as a result of communicative merge, the offer or judgment is realization of informational requirement.

\subsection{Intrinsic Neuronet}

Let $\mathrm{S}$ - the spelling dictionary, where $\mathrm{S}=\{\mathrm{Si}\}, \mathrm{Si}-\mathrm{a}$ morphological word. The word Si calls sign of Qij of the representative of $\mathrm{Mij}$ from a set of $\mathrm{Mi}$ where $\mathrm{Mi}=\{\mathrm{Mij}\}$. Let's designate a lexical meaning of the word Si through \{ Mij, Qij, Si \}. We will set communication of lexical meanings of words $\{\mathrm{Si}\}$ with elements of a set Mi set of the relations of signs of Qi where

$$
\mathrm{Qi}=\{\mathrm{Qij},(\mathrm{Mi}, \mathrm{Mij})\} .
$$

The set of lexical meanings of the relations of signs with 
representatives connected by set is an intrinsic neuronet. Words in a neuronet are supplied with indexes of signs according to their relations of signs with representatives. The intrinsic neuronet fixes substances of signs of representatives. The dictionary helps to use words with the lexical meaning and to distinguish representatives whom they call.

Words are used on a basis the indexes of signs. Each sign has three indexes. One index indicates subject domain of knowledge, the second a situation, the third for the situational moment. Words with several lexical meanings, have several sets of indexes. For example, flour and flour. The word the field is used in various subject domains. Each set of indexes defines a lexical meaning of a word.

The intrinsic network of language thinking operates with intrinsic elements of knowledge: words, offers, judgments. Shock words in the offer are marked with two strokes. For example, *I* was in China. I *was* in China. I was in *China*.

The software imitating work of neuronic network includes the following sequence of actions:

a) Modernization of neuronic network in the user's parameters, or use established by default.

b) Tutoring of network.

c) Message to the user of the answer.

The intrinsic recursion neuronet with a reinforcement studies and functions on the basis of weights of meanings of elements of knowledge of subject domains.

\subsection{Definition of Grammatical Types and Signs of Knowledge Elements}

Actual and abstract representatives have signs of change, space, an object, the subject, action, quality, quantity etc. They are put in language into words according to particular grammar rules.

Definition 1. The grammatical characteristic of a set of words of category of signs which morphological value is formed by the common rules of grammar is called a grammatical look.

The noun, adjective, verb, adverb, pronoun, participle, numeral, adverbial participle and interjection are grammatical types of Russian.

Words which are formed in language from a word with a principal value are congenerous. For formation of congenerous words the prefix before the main word and a suffix after a basis of the main word serves. The terminations depend on a suffix.

Definition 2. The set of words of one grammatical look, congenerous to a word with a principal value, is called informational sort.

Definition 3. Two sorts of a different grammatical look are called congenerous if their words with principal values congenerous.

Definition 4. The set of congenerous informational childbirth is called family.

The family contains the congenerous words of different grammatical types. In families congenerous words are ordered by types and in a look.
Definition 5. The set of the words forming phrases with the set is called its informational community.

The number of representatives of community of a word defines its semantic intensity.

Theorem 1. Any offer can be created on its syntactic structure from a necessary and sufficient set of phrases by morphological, syntactic and their semantic analysis and merge.

Definition 6. It is related the offers merged according to contents through word forms of families and is associative the offers merged according to contents we will call the partial associative judgment.

Definition 7. The sequence of offers $\mathrm{P} 1, \ldots, \mathrm{Pn}$ is called associative judgment if for all $\mathrm{i}, \mathrm{Pi}, \mathrm{Pi}+1$ is the partial associative judgment.

Theorem 2. Any partial associative judgment of $\mathrm{C} 0$ it is possible to create on its syntactic structure $\mathrm{CC}$ of necessary situational and valence offers by morphological, syntactic and their semantic analysis and merge.

Theorem 3. Any associative judgment of $\mathrm{C} 0$ it is possible to create on its syntactic structure $\mathrm{CC}$ of necessary partial associative judgments by morphological, syntactic and their semantic analysis and merge.

\subsection{Subject Domains of Knowledge}

Subject domains of knowledge represent networks is associative the bound informational requirements and their realization from communicative bound symbolical language elements (informational traces of communicative and associative symbolical and language thinking). Realization of informational requirement can be itself informational requirement. Then associative to it the network of communicative bound elements will be its realization. The combined informational requirements consist or of the enclosed informational requirements of subject domain of knowledge, or of the sequence of informational requirements, or of the sequence of the enclosed informational requirements. The combined informational requirements consist either from prime, or from prime and combined, or of combined. Informational requirements of subject domain of knowledge form combinational expansible network.

The set of elements of knowledge with a marking is a knowledge base. Imperative or the interrogative sentence of a knowledge base is informational requirement. The set of procedures of realization of informational requirement is base of abilities.

According to theorems 1-3, informational requirement has realization in a knowledge base and base of abilities if there is a necessary and sufficient set of elements of knowledge in a knowledge base and procedures of realization in base of abilities.

For the informational requirement which is a combination of informational requirements for which there is a realization there is a necessary and sufficient set of elements of knowledge in a knowledge base and procedures of realization in base of abilities. The corresponding combination of necessary and sufficient set of the available realization will 
be realization of the combined informational requirement. The symbolical and language communicative logic connects the realized informational requirements, that is the true.

Let CS - the intrinsic dictionary of subject domain of knowledge be set;

BIP - the basic informational requirements of subject domain of knowledge made of the words of the CS dictionary. Basic informational requirement is either the voprosny, or imperative offer;

RBIP - realization of basic informational requirements;

PKIP - rules of combination of informational requirements from basic.

KKASRIP - the designer of communicative and associative network of realization of informational needs of the user;

KASRIP(b) - communicative and associative network of realization of basic informational requirements of subject domain of knowledge.

Let the user it is set (IPi), made by the rules PKIP. Let's apply to it the designer of KKASRIP. Let's receive KASRIP(i) expansion of KASRIP (b) network with realization (IPi).

The realization of informational need a question for communicative and associative network of subject domain of knowledge is enabled as follows. Let the offer P1 with a marking be informational requirement a question with a situational scheme of signs SP1 from the situational scheme $\mathrm{S} 1$. The word a question in the offer $\mathrm{P} 1$ is defined to not described sign of Q1 of a lexical meaning of LZ1 of informational niche of IN1 is known.

It is necessary to find in communicative and associative network of subject domain of knowledge the offer P2 containing phrases and lexical meanings of the offer P1 and the lexical meaning of LZ2 which is the description of sign of Q1.

The procedure of realization builds according to the offer P1 the R2Sh template with not described sign of Q1 of the offer P2. On the R2Sh template, its marking, the SP1, S1 procedure of realization looks for the offer $\mathrm{P} 2$ in network of communicative phrases of subject domain of knowledge by means of procedures of the morphological, syntactic and semantic analysis. The found offer P2 will be realization.

Imperative informational requirement for searching of its realization is replaced with the equivalent informational requirement a question.

Conclusion: language communicative logic of technological thinking algorithmically solvable.

\section{Specialization of the Android}

Specialization of cognitive robots is carried out on the basis of knowledge bases, bases of abilities and implementers of behavior.

The knowledge base contains data domains of knowledge.

Data domains of knowledge contain the intrinsic oriented dictionaries. Communicative words of the intrinsic oriented dictionaries generate phrases, communicative phrases generate sentences, communicative sentences generate opinions. Information needs are imperative and questions.

They it is associative are connected in data domain of knowledge to the implementations. Implementation can be itself information need.

Data domains of knowledge represent networks communicatively and is associative the coupled symbolical language elements with a situational and character and language marking.

The network $\mathrm{G}(\mathrm{Xt}, \mathrm{Gt}, \mathrm{Vt})$ is conceptual representation of knowledge if $\mathrm{Xt}=\mathrm{U} \mathrm{U}$ where $-\mathrm{a}$ set of peaks of opinions with a situational and language marking, - a set of peaks of the sentences having a language and situational and character marking: $\{($ a lexical meaning, a look), (a sign, a niche $)\},-$ a set of peaks of phrases with a character and language marking, $\mathrm{Vt}-\mathrm{a}$ set of communicative communications of set members of $\mathrm{Xt}, \mathrm{Gt}$ - a set of the associative communications of set members of $\mathrm{Xt}, \mathrm{t}$ - the discrete moments of appearance of new elements of knowledge, a sign - the sign of the representative of a reality specifying the semantic use of the word in the speech and in writing a look - the grammatical characteristic of a set of words which morphological value is created by the general rules of grammar a niche - uniform situational use of a set of words of different types in different syntax sentence structures (the niche can be subject and object, procedural, a circumstance, attributive, additional.

The associative communications connect peaks which are information need and its implementation. Communicative communications proceed from peaks of communicative elements of knowledge of which phrases of a sentence and an opinion are created.

The labeled morphological words of the information job of the person to the robot can be either a lexical meaning of the semantic oriented dictionary, or analytical action, or behavioral action. For example, $<$ to add $>$, there can be a lexical meaning, behavioral action (to put things) or arithmetical action $(+)$. Let's consider network structure of addition of two numbers 10 and 15 .

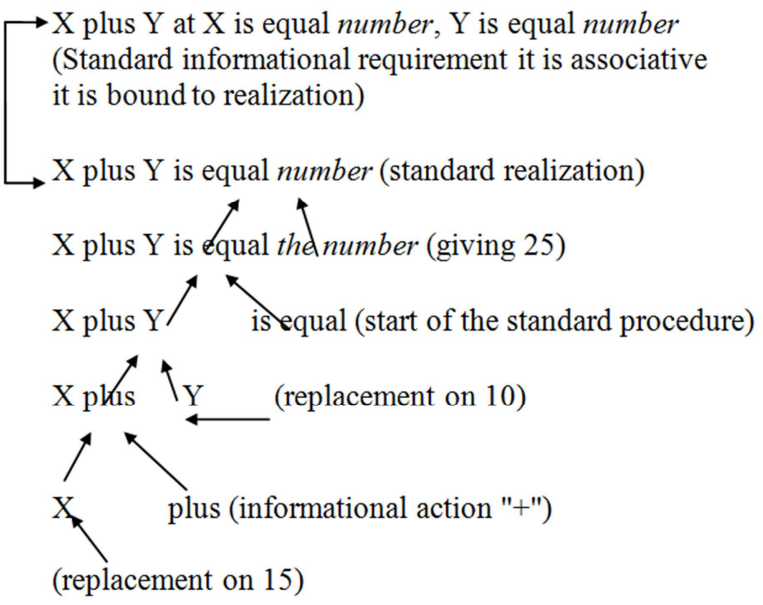

Figure 1. Network structure of addition.

The compulsory provision of feasibility of information 
requirement is completeness of the knowledge base and base of abilities, that is existence of necessary and sufficient set of elements of knowledge and abilities of cross-disciplinary subject domain for training in a healthy lifestyle.

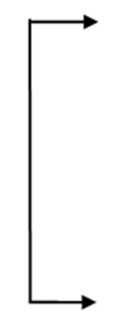

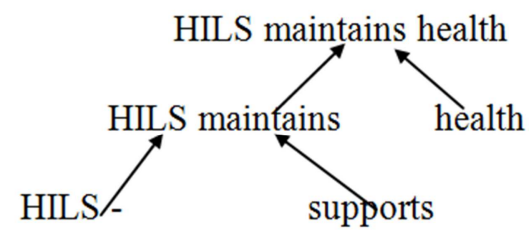

The healthy lifestyle is carried out by the useful habits

The healthy lifestyle is carried out by the useful
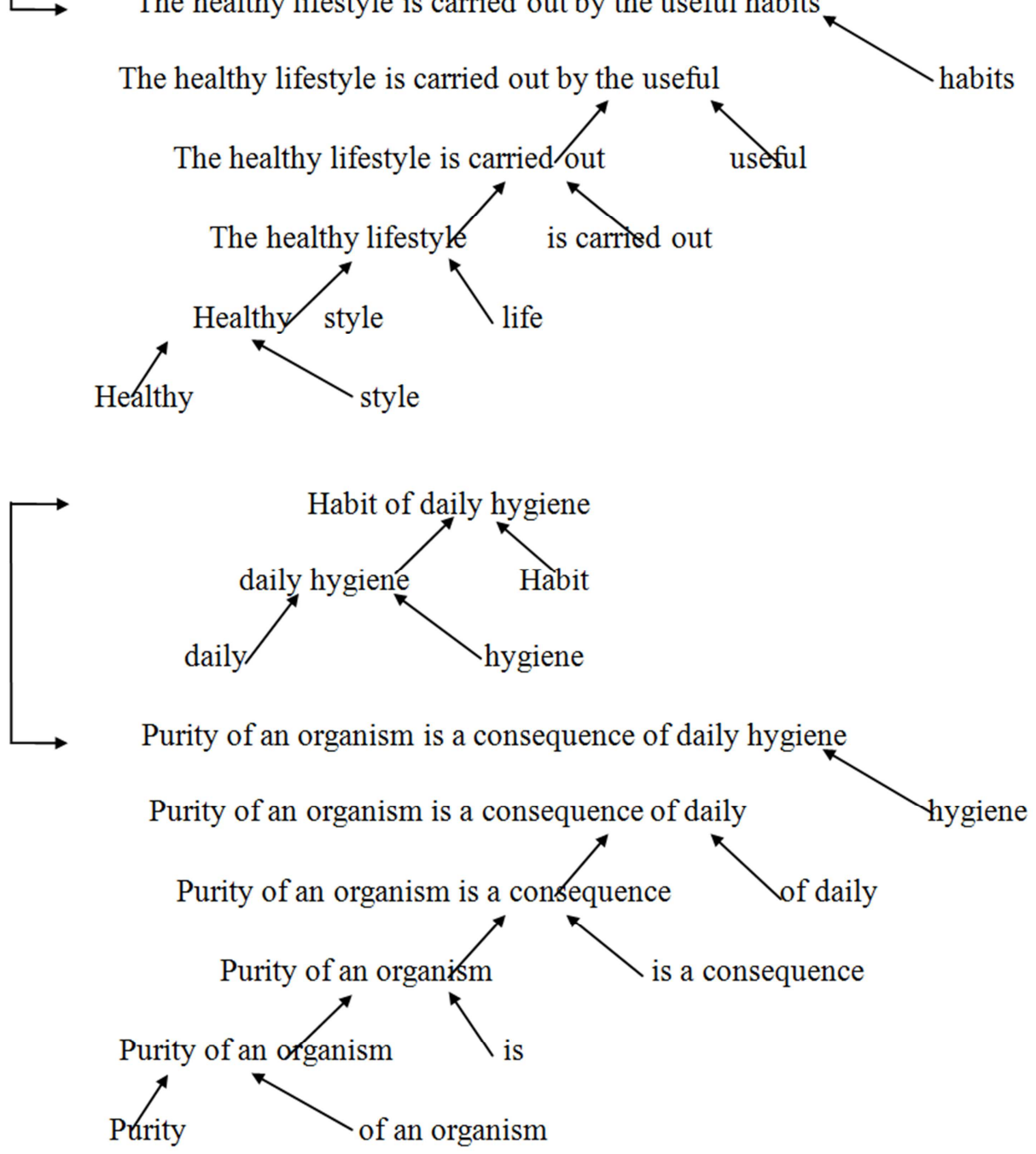

Figure 2. Communicative-associative network structures.

The healthy lifestyle of the person is under construction on the basis of high-quality regularities. High-quality regularities are described by causes and effect relationships. The communicative and associative network of knowledge of the cross-disciplinary subject domain concerning a healthy lifestyle contains causes and effect relationships between elements of knowledge describing useful effects and the corresponding improving investigations generated by them. The realization of a healthy lifestyle is enabled by useful habits. Purity of an organism is reached by a habit of daily hygiene.

Consultation of the robot in communicative and associative network of elements of knowledge of subject domain is based as a chain of relationships of cause and effect. For example, in the mode short consultation: (a habit daily hygiene) - (purity of an organism).

Realization of information requirement can be information requirement. In this case on a communicative tree of lexical meanings of the reason by their synthesis we reach associative top. Then we determine realization by associative communication.

The robot can train along with consultations in physical exercises. To physical culture for normalization of a tone of an organism. To gymnastics for normalization of rhythms of functioning of an organism. To charging on normalization of a power system. 
With the knowledge base several users can interact. For them the administration directed to coordination and the accounting of different requirements, and the conflict resolution is entered. The administrative subsystem of management system of the knowledge base services its users and their and current requirements. Appearing as the intermediary between users, she will agree on appeals to elements of knowledge of data domain, and also input of new elements of knowledge. Standard information needs of users and their standard implementations are included in the knowledge base.

The management system the knowledge base will organize direct access to elements of knowledge in implementation process of information need and for development of the knowledge base new implementations.

The management system transfers by the knowledge base to a subsystem of language communication either implementation of information need of the user, or the message on absence of knowledge for its implementation.

The basis of abilities contains standard procedures of implementation of standard information actions, procedures of implementation of combined information actions, procedures of implementation analytical (mathematica, chemical and others) actions and the procedure of implementation of behavior of the robot.

The robot component realizing the adaptive behavior in the external environment includes sensor, controlling, executive motor systems and system of diagnostics [8-19].

The sensor system is intended for perception and information transform about a status of an external environment. It turns on television and optiko-laser devices, ultrasonic range finders, tactile and contact sensors, situation sensors, neural network devices of image identification of an external environment, etc.

The system of diagnostics exercises control of execution of simple movements of parts of motor system of the robot on each step of implementation of behavior, transmission of necessary information of the managing director to system about a status of motor system for its correlation with information from sensor system in real time, and also reports about the end of simple movements.

The executive motor system realizes procedures of behavior of the robot in the external environment realizing various movements. The motor system has mechanical hands (pointing devices), mechanical legs (pedipulators). The pointing device can take, turn, transfer, collect, bend hindrances, etc. The legged robot can move on unfamiliar terrain with a difficult relief, overcoming hindrances.

The procedure of implementation of behavior is built on type a situation action. In the mode of implementation of behavior the controlling system permanently processes information on a situation from sensor system and from system of diagnostics and launches executive system.

The robot determines model of the external environment by information need of the person and touch information. He determines behavior model by model of the external environment and information need of the person. Then he determines the sequence of the behavioural acts of motor system in the external environment on type a situation action realized from the point of view of the functional and touch opportunities by models of the external environment and behavior.

For a certain external environment at the robot the model of the external environment, behavior model and procedures of realization of behavior are set. In a certain external environment the robot on the information need of the person (INP), models of the external environment, behavior model realizes information need of the person procedures of realization of behavior. On IPCh the robot forms network of bit-by-bit realization of information requirement for subject domain. If the network is built, then the imitative thinking starts procedures of realization of IPCh. In the course of realization of IPCh the robot via touch devices controls a condition of the external environment. If the condition of the external environment on any circumstances doesn't correspond to a condition of model of the external environment, then the robot gives the message that it can't realize IPCh in connection with change of the external environment and waits for the following information requirement from the person. After realization of IPCh the robot reports to the person about the termination and results of the activity.

In the uncertain external environment the robot according to touch information forms model of the external environment (EE), selects standard behavior model for IPCh and consistently realizes IPCh procedures of realization of behavior: lays a safe route of the movement of the robot on the VS model, on behavior model the standard procedure builds the serial-parallel movements of manipulators, pedipulyator and other motor mechanisms of the robot.

The robot for work with the uncertain external environment has many various touch devices. The behavior model of the robot forms the procedure of realization of IPCh of a set of standard procedures. The behavior of the robot is implemented along a route of the movement and the sequence of intermediate configurations of the motive acts leading to realization of IPCh. The sequence of configurations of motive acts is built according to a route and IPCh.

The behavior model builds movements of motor mechanisms of the robot under the law of change of their generalized coordinates guaranteeing implementation of IPCh. Movement is determined by the vector of the generalized coordinates determining the current provision of degrees of mobility of its mechanical part. Working parts of motor mechanisms make rotations on the trajectory determined by a vector of phase coordinates. Interaction with the external environment and its perception performs the robot by means of different sensors and touch systems. Real situations are described in memory of the robot by means of a set of indications of touch sensors. Touch information can be photographic, scanned, range - metricfrom optical and ultrasonic systems of technical sight.

In terms of indications of touch sensors primary 
description of model of the external environment is created. The analysis and handling of this information lead to the generalized description of a situation with the help of concepts. According to the generalized description of a situation and IPCh the behavior model of the robot and a set of standard procedures of implementation of IPCh are selected.

In behavior model functional properties of the robot, mobile opportunities of the robot are reflected in the external environment. Thanks to it the robot can render services in IPCh, according to mobile opportunities, the VS model and behavior model in the unfamiliar external environment.

The task of the analysis of touch information, recognition and the description of a situation is urgent for interaction of the robot with the uncertain environment. This problem by training of the robot is solved to distinguish objects of the external environment through systems of recognition of threedimensional objects, to describe in a natural language elements of three-dimensional scenes on the basis of touch information by means of a full range of elementary situational signs. For example: further, more to the left, below, are disconnected, areas of different color, etc. The description of the external environment is set in a natural language by means of a full range of elementary situational signs.

The robot, analyzing a situation of the external environment on standard model of the external environment, touch information, to the description of a scene of the external environment selects for IPCh behavior model, procedures of implementation of behavior, builds a route of movement and consistently creates motive acts of parts of motor mechanisms of the robot for requirement implementation. Differentiate cyclic and position systems of coordination of motive acts. In cyclic systems of a trajectory of motive acts are limited to 2-4 points of positioning on each of mobility degrees, and in position systems the number of these points can constitute several tens that allows to implement are difficult" e motive transactions.

Cyclic and position management provides movement of working body for a broken trajectory from a point to a point.

The concept of relative mobile system of coordinates is central. Relative mobile systems of coordinates are connected with each position point of a part of the motor mechanism.

For each position point on each step is defined consistently at what size and in what direction to move her from the current situation to set. For each part of motor mechanisms connected with a position point is defined in what direction and on what corner it is necessary to turn.

Simultaneous turn and transfer of a part of motor system concerning the generalized system of coordinates are calculated on formulas [6].

For pneumatic parts of motor mechanisms it is set to increase or reduce the scale specifying in how many time the part size. The behavior model and procedures of realization of behavior are defined by the field of professional activity in which the robot has to realize IPCh.

The directing cosines of part P1P2 of motor system of the robot in own relative system of coordinates it is calculated on formulas [6].

The behavior model contains algorithms of adaptive integrated management of the movements, a route of movement of the robot and the sequence of motive acts of parts of motor mechanisms of the robot.

Procedures of realization of behavior are performed by the movements of parts of motor mechanisms of the robot according to a route of movement and the sequence of motive acts of parts of motor mechanisms of the robot. The route of the movement of the robot is under construction in classes of piecewise and polynominal functions.

Let the area represent the plane, obstacles by broken lines and coordinates of a starting point of the robot and target where the robot has to move are set.

It is necessary to construct an optimum route as the broken line from a starting point in target which doesn't cross obstacles and has the smallest length.

This problem is solved by a classical method of dynamic programming of consecutive approximations in functional space, using a recurrence relation of function $f$, the defining route length from a starting point target. The strategy of finding of the minimum route is defined by consecutive approach of the constructed any route to minimum.

After the optimum, safe route is constructed, by methods of the generalized, relative and phase coordinates motive acts of motor parts of the robot are under construction.

Coordination of motive acts is consecutive - information coordinator of behavior model which is in the field of attention of memory of the robot in parallel carries out.

\section{Management of Behavior of the Android}

Behavior of the created robot the multifunctional hierarchical system of controllers, similar to a control system of behavior of a human body operates. The behavior arises under the influence of informational requirement which causes orientation in a situation on models of the external environment and behavior. Orientation comes to the end with the choice of the corresponding line-up of programs of hierarchical system of controllers as instructions to actions which realization leads to realization of behavior. Advantage of the organization such consists in development of behavior of the robot on the basis of expansion of hierarchy of the subprogrammes setting acts of behavior.

The controller is a computer on a chip. It is intended for control of various electronic devices. The controller of the robot is the diminutivest computer. It contains the processor and peripheral devices: FLASH memory, timers, interfaces for communication with peripheral equipments and a set of other useful schemes. The controller operates according to the given program which is loaded into it from the potent computer. It is loaded by means of a programmator (in the simplest look is an express cable) into the microcontroller.

The robot has bodies for interaction with a surrounding 
medium. Bodies which obtain information from a surrounding medium are called receptors (or sensors). And bodies which influence a surrounding medium - effectors: engines, loudspeakers, light-emitting diodes and so forth. The controller has a quantity of entrances and exits. To entrances receptors, and join exits effectors. Microcontrollers can process information from entrances and create electric signals at the exits how we will program behavior of the robot [6-7].

\section{Robotic Smart Drugstores}

Androids with technological thinking, such as Sophia or Bina48 of the Hong Kong campaign Hanson Robotics Ltd, the Japanese robot ASIMO, the Russian robot Alice it is possible to train and use as sellers of clever cafes or drugstores [8-11]. Visitors of smart drugstores are served by the robot and the automatic machine of piece drugs. The robot has the neural network trained system of communication with visitors for booking, the device of reading of electronic money from the plastic card of the visitor and the device of distance steering by the program controller of the automatic machine for delivery of drugs. The automatic machine of delivery of products under control of the robot serves orders of visitors. The visitor inserts the plastic calculated card into the device of reading of electronic money, reports to the robot the list of drugs of the order. The robot through the system of booking and the program versatile controller of the automatic machine activates trays with drugs according to the order. When the order is created by automatic machine for delivery of drugs, the robot reads out electronic money from the plastic card of the visitor, at the prices of drugs. After the visitor receives a set of drugs of the order from the automatic machine, takes away the calculated plastic card and the check.

\section{Conclusion}

Use of programmable production will demand the universal mobile robots capable not only to carry out in advance set of operations in a workplace, but also to move freely on production rooms, to transfer between jobs of a component and finished products and to react flexibly to changes in production. Soon such physically simple affairs as work of the druggist or librarian will be sent to a bookdepository to robots.

A large number of almost completely robotic factories and plants can appear by 2020 . Robots will begin to use actively in agriculture and also the specialized robots helping the person with hard physical activity. On streets of our clever cities we will see robots cleaners, robots loaders, robots of trishaws and of sellers.

\section{References}

[1] Evgeniy G. Bryndin, Symbolical and language communicative and associative technology of imitative thinking. Inter. Conf. "High technologies, basic and applied researches, education". S-PB: PSTU Page 442-444. 2007.

[2] Evgeniy G. Bryndin, Theoretical bases of communicative and associative imitation of symbolical and language thinking. J. Information technologies. N. 2. Page 29-34. 2009.

[3] E. I. Yurevich, Intelligent robots. Publishing house: Mechanical engineering. 360 pages. 2007.

[4] Evgeniy G. Bryndin, The robot with communicative and associative imitation of symbolical and language thinking. Inter. Conf. "Intellectual tekhnolgiya in education, economy and management". Voronezh: ВИЭиСУ. Page 429-439. 2010.

[5] Evgeny Bryndin. Bases of imitation of thinking and continuous processing of programs. Germany: LAP LAMBERT Academic Publishing. 197 pages. 2012.

[6] Evgeniy G. Bryndin The Robot with imitative thinking. "PNIPU bulletin: Electrical equipment, Information technologies, Control systems", N. 14. Perm: PNIPU. Pages 536. 2015.

[7] Evgeny Bryndin. Control of the robot with imitative thinking. Germany: LAP LAMBERT Academic Publishing. 77 p. 2015.

[8] Evgeniy Bryndin. Program Hierarchical Realization of Adaptation Behavior of the Cognitive Mobile Robot with Imitative Thinking. International Journal of Engineering Management. Volume 1, Issue 4. 2017, pp. 74-79.

[9] Evgeniy G. Bryndin, Cognitive robot consultant for a healthy lifestyle. III International scientific conference "Information Technologies in Science, Management, the Social Sphere and Medicine". TPU. Pages 484-488. 2016.

[10] Bryndin E. G. Cognitive robots. Inter. Conf. "Management of development of large-scale systems (MLSD'2016). M.: IPM RAS. Pages 285-294. 2016.

[11] Evgeniy Bryndin. Cognitive Robots with Imitative Thinking for Digital Libraries, Banks, Universities and Smart Factories. International Journal of Management and Fuzzy Systems. V. 3, N. 5, 2017, pp 57-66. 